\title{
Review Board (Vol. 10 No. 1)
}

Avril Aitken, Bishop's University

Susann Allnutt, Education Consultant

Ramona Arora, University of Texas at Austin

Natasha Artemeva, Carleton University

Maggie Baden-Smith, University of

Worcester

Dale Boyle, McGill University

Vera Caine, University of Alberta

David Dillon, McGill University

Margaret Dobson, Education Consultant

Robert Donmoyer, University of San Diego

Lynn Driedger-Enns, University of Alberta

Linda Furlini, McGill University

Karen Gazith, McGill University

Limin Jao, McGill University

Catherine Le Maistre, McGill University

Lerona Lewis, McGill University
Warren Linds, Concordia University

Anne Murray-Orr, St. Francis Xavier University

Stephanie Pinnegar, Brigham Young University

Gail Prasad, University of Wisconsin

Debbie Pushor, University of Saskatchewan

Caroline Riches, McGill University

Carolyn Sturge Sparkes, Memorial University

Tamara Sussman, McGill University

Lisa Trimble, McGill University

Andrea Videtic, Champlain College

Boyd White, McGill University

Lee Williams, Slippery Rock University

Paul Zanazanian, McGill University 